\title{
Impacts of climate change on soybean production under different treatments of field experiments considering the uncertainty of general circulation models
}

\begin{abstract}
ABSTRCT
Earth is faced with dramatic changes in the weather systems, which leads to climate change. Climate change affects water resources and crop production. In this study, five and seven general circulation models (GCMs) were respectively collected via the IPCC Fourth and Fifth Assessment Reports. Emission scenarios including B1, A1B, and A2 for AR4 and RCP2.6 and RCP8.5 for AR5 were applied to predict future climate change. The weighting method of mean observed temperature-precipitation (MOTP) was utilized to compute uncertainty related to different climate models. The scenario files made by $\Delta \mathrm{T}$ and $\Delta \mathrm{P}$ were applied to the downscaled model of LARS-WG to generate weighted multi-model ensemble means of temperature and precipitation for the period 2020-2039 centered on 2030s. These ensemble means were incorporated into the calibrated AquaCrop model to predict final yield and biomass. In this study, soybean data were applied for four different varieties under three irrigation treatments in field experiments carried out at Karaj Seed and Plant Improvement Institute in two successive years. However, the results of statistical analysis between the model output and observed data for all varieties and irrigation treatments in the calibration year (2010) and validation year (2011) were the same at the $95 \%$ confidence level. It is suggested that AquaCrop is a valid model to predict yield and biomass for the study area in the future. Furthermore, comparing future climatic variables to the historical period during the soybean growing season showed enhancement of these variables by the 2030s. The amplitude change of temperature was larger in AR5, whereas the amplitude change of precipitation and $\mathrm{CO} 2$ were larger in AR4. The soybean yield and biomass increased for all treatments in the $2030 \mathrm{~s}$ with positive correlation with the climatic variables. The maximum temperature represented the most significant correlation with yield and biomass for almost all treatments. Finally, soybeans might achieve an optimal threshold temperature in the future, leading to yield increases in the 2030s.
\end{abstract}

Keyword: AquaCrop; Yield; Biomass; GCMs; Emission scenarios. 